\title{
INDIVIDUALISME ET STATISTIQUES
}

Michel Bourdeau (IHPST-CNRS-PARIS1)

Olivier Rey : Quand le monde s'est fait nombre; Paris, Stock (Les essais), 2016, 322p.

« Les nombres gouvernent le monde » disait Pythagore ; mais il pensait au seul monde physique et il a fallu attendre le dix-neuvième siècle pour songer que cela pouvait aussi s'appliquer au monde social. Comment en est-on venu là ? Signe de l'importance de la question, plusieurs ouvrages excellents lui ont déjà été consacrés, par Alain Desrosières, Eric Brian, Ted Porter et dernièrement encore par Alain Supiot. Le livre d'Olivier Rey ne fait double emploi avec aucun d'entre eux. Bien que le fil suivi soit chronologique, il ne s'agit pas d'une histoire des statistiques, ni même de ses usages sociaux. Le propos est plus ambitieux : expliquer cet essor des statistiques en le mettant en rapport avec les profondes mutations qu'ont connues nos sociétés au cours des derniers siècles. L'enjeu est loin d'être seulement historique puisque, par ce biais, c'est une explication de notre temps qui est visée : « trouver un point de vue global sur nos mondes éclatés » (P.15-16).

Après une brève introduction, qui précise l'objet du livre et ses méthodes, l'ouvrage commence par décrire comment, pour les besoins de leur administration, les États modernes, en France avec Bodin, en Allemagne avec la caméralistique, se sont trouvés amenés à récolter toute sorte de données, qui n'étaient pas nécessairement quantitatives, sur la situation du pays. L'Angleterre, déjà, se distinguait en ce que ce ne fut pas l'État, mais des individus, Graunt, Petty, qui fondèrent l'Arithmétique politique. Et dès cette époque, le nombre, c'est d'abord le nombre des hommes.

Le chapitre trois passe alors de la description à l'explication et présente une des thèses centrales du livre : le succès des statistiques est dû à un changement dans les rapports entre les êtres humains, plus précisément, à un changement dans le statut de l'individu. Jusqu'alors, conformément à l'étymologie, il s'agissait d'un concept négatif, comme atome, in-sécable : l'individu n'était que le terme dans la suite des divisions de l'arbre de Porphyre. Désormais, avec Descartes, il devient au contraire point de départ. Cela induit corrélativement un changement dans notre conception de la société. C'est le passage décrit par Tönnies de la communauté (Gemeinschaft) à la société (Gesellschaft). Entre les statistiques et cette nouvelle conception de la société, il existe comme une complicité originaire : il n'est plus nécessaire de faire appel à un principe organisateur puisque, lorsque l'on part de l'individu, on voit l'ordre surgir spontanément. Mais, si les concepts se mettent en place dès le $17^{\mathrm{e}}$ siècle, pour qu'ils s'incarnent, prennent chair, il faut lever les obstacles qui s'opposent à leur réalisation. Il apparaît alors que l'obstacle majeur, c'est l'Ancien Régime lui-même, qui aurait bien voulu développer d'avantage les statistiques mais se heurte à ses propres limites. D'où la nécessité de révolutions, politiques et industrielles. Pour instaurer la société des individus, Il faut détruire tout corps intermédiaire comme corporation ou association. Seuls existent l'intérêt particulier et l'intérêt général ; entre, il n'y a que des factieux. Mais l'essor des statistiques tient encore à leur fonction spéculaire. Pour penser le nouveau type de société en train de se mettre en place, aucun cadre conceptuel n'est disponible et l'individu, en même temps qu'il s'autonomise, ne comprend pas le monde dans lequel il vit. Les statistiques lui offrent une grille d'analyse. Elles ne servent d'ailleurs pas seulement à décrire une réalité préexistante, elles contribuent aussi à transformer le monde : qu'on pense à l'impact du classement de Shanghai sur la politique des universités.

Suit un chapitre à nouveau essentiellement descriptif. L'engouement pour les statistiques est tel qu'on en trouve des traces jusque sur la scène (Labiche, Offenbach). Pendant ce temps-là, la résistance s'organise dans le monde savant : chez les économistes, 
qui y voient une potentielle rivale, qui plus est au service de l'État qu'ils combattent, mais aussi chez les médecins attachés à la clinique.

Pour expliquer l'essor prodigieux des statistiques au dix-neuvième siècle, il ne suffit toutefois pas d'invoquer la place nouvelle accordée à l'individu, il faut aussi considérer un phénomène qui en est comme l'envers : la question sociale. La société apparaît en effet rongée par un mal imprévu. Les progrès de l'industrie étaient censés apporter bonheur et prospérité. Mais si cela vaut pour une partie de la population, pour une autre partie, bien plus nombreuse, c'est tout le contraire. Pour étudier le phénomène, le mieux est de s'en tenir au cas anglais car c'est en Grande-Bretagne qu'il a pris sa forme la plus aigue. Le tableau dressé par les voyageurs, que ce soient Tocqueville ou Engels, est accablant. La brutalité du processus n'a rien à envier à ce qu'a été la colonisation et dès 1755, Benjamin Franklin estimait que « le travail des esclaves [en Amérique] ne peut jamais être aussi bon marché que le travail des ouvriers en Grande-Bretagne ». Comment cela était-il possible, dans le pays alors le plus avancé économiquement et politiquement? Les débats se concentraient autour de la loi sur les pauvres, qui assuraient une protection sociale aux plus démunis et que ses adversaires accusaient de perturber la bonne marche de l'économie. Comme on le verra, c'est dans ce contexte que s'inscrit l'œuvre de Malthus mais, indépendamment de la réponse théorique apportée par celui-ci, la gravité du phénomène appelait une action immédiate. C'est ainsi que la question sociale a puissamment contribué au développement de la statistique. En France, par exemple, ce sont les grandes enquêtes médicales et sanitaires de Villermé, qui visaient à mieux connaître le mal pour le combattre plus efficacement.

Avec les deux chapitres suivants, on change de registre : il s'agit non plus directement des phénomènes sociaux, mais du rôle des statistiques dans la constitution des sciences. L'histoire, ici, met à mal une idée reçue sur les rapports entre sciences de la nature et sciences sociales, qui veut que celles-ci imitent celles-là. En l'occurrence, c'est le contraire qui s'est passé, puisque c'est avec les sciences sociales que les statistiques ont fait leur entrée dans le champ théorique. L'initiative en revient à Quetelet, le théoricien de l'homme moyen, qui constate que les mesures des conscrits se répartissent selon la loi des erreurs (la fameuse loi de Laplace Gauss, la courbe en cloche) établie par les astronomes. Cette découverte des régularités statistiques, qui assure la liaison entre statistiques et probabilité, est un événement dont on a du mal à mesurer à quel point il a frappé l'imagination des contemporains. De cette « forme merveilleuse de l'ordre cosmique » qu'est la loi normale, Galton, le cousin de Darwin, n'hésitait pas à dire qu' " un sauvage, s'il pouvait la comprendre, la vénérerait comme un dieu » (p. 178). Ceci étant, très tôt, deux problèmes se sont posés. L'un politique : si les phénomènes sociaux obéissent à des lois, quel sens y a-til à prétendre gouverner une société ? Autrement plus embarrassant est le problème moral, lié à l'émergence de concepts appelés à une fortune exceptionnelle, ceux de norme et de normal. La loi normale ne se contente pas d'énoncer un fait, de dire ce qui est, elle définit aussi la norme, ce qui doit être. Quoi de plus équivoque que le concept de norme, qui glisse sans cesse de l'être au devoir être, du descriptif au normatif ? De la stabilité des statistiques des crimes ou des suicides, quoi de plus tentant que de conclure qu'il s'agit là de phénomènes normaux ? On connaît la réponse de Durkheim : il faut distinguer le type moyen (celui dont parle Quetelet) du type collectif, qui est du domaine de la représentation et dont l'accès échappe à la statistique. D'abord conçu comme mode d'appréhension de l'autonomie du social, il s'avère que celle-ci souffre d'une cécité de principe aux manifestations les plus essentielles du social.

Du chapitre suivant, remarquable par la clarté avec laquelle sont exposées des théories difficiles, on retiendra surtout deux points. Tout d'abord, la résistance des scientifiques à l'introduction des statistiques, qui choquaient leur croyance au déterminisme. Il faudra donc que ce soit le père de la physique sociale, Quetelet, qui montre au physicien, 
Maxwell, la voie qui conduisait à la théorie cinétique des gaz. En second lieu, l'auteur souligne, point souvent négligé par les épistémologues, la contribution de premier plan de la génétique et de la théorie de l'hérédité au développement de la statistique mathématique. Ainsi, c'est à Galton que l'on doit la notion de régression et, si elle porte ce nom, c'est qu'elle lui servait à décrire ce qu'il considérait comme des phénomènes de dégénérescence.

Le succès des statistiques ayant trouvé un écho jusque dans la littérature, un dernier chapitre lui est consacré, ce qui nous vaut par exemple quelques pages sur l'aptitude respective de Balzac et de Stendhal à rendre compte des phénomènes de foule. Face au nombre, les écrivains ont en effet adopté les attitudes les plus diverses. Certains ont reconnu l'utilité des informations qui leur étaient ainsi fournies, tandis que d'autres tournaient en dérision leur futilité, quand ils n'affichaient pas, comme Baudelaire ou Joyce, leur mépris pour la multitude et tout ce qui trouve une expression numérique.

Le sens de la conclusion est tout entier dans son titre : hainamour statistique. La statistique n'est pas aimée. Impersonnelle, elle aplatit tout et l'ego souffre de n'être plus qu'un simple élément, perdu au milieu d'une masse d'anonymes comme lui. De plus, elle est trompeuse, puisque ce qui nous est présenté comme une donnée indiscutable est en réalité le fruit d'une construction savante, passée sous silence. Notre défiance est encore renforcée par l'usage qu'en font les hommes politiques, à qui elle permet de prendre des décisions sans donner l'impression d'en prendre. Mais dans quelle mesure leur rejet est-il justifié? Des statistiques, on attend deux choses incompatibles : Rendre fidèlement compte d'une réalité complexe, tout en en donnant une image simple, facilement intelligible. Notre défiance elle-même est contradictoire dans la mesure où l'essor des statistiques est indissolublement liée à des conditions sociales auxquelles nous ne sommes pas prêts à renoncer.

Le grand mérite d'Olivier Rey est d'avoir mis en évidence, comme personne ne l'avait fait avant lui, cette ambivalence de notre rapport au nombre. Parler de succès serait en effet simplifier de façon abusive la situation et l'on serait tout aussi bien en droit de parler d'échec. Certes, les statistiques sont omniprésentes ; mais cette donnée objective ne dit rien sur la façon dont est vécu cet empire du nombre. Dès le moment où ce second aspect est pris en compte, le tableau change. S'il se sait soumis aux statistiques, l'homme moderne répugne à se reconnaître dans le miroir qu'elle lui tend et se méfie d'elle. Le problème se dédouble donc : expliquer la place de la statistique dans nos sociétés, ce n'est pas seulement expliquer leur omniprésence, c'est aussi expliquer le rapport ambivalent que nous entretenons avec elle. A cette dernière question, qui n'est pas moins importante que la première, Olivier Rey répond : les statistiques ont ceci de remarquable qu'elles mettent l'individu face aux contradictions de l'individualisme (p. 297). Le concept d'individualisme étant notoirement obscur, il ne sera pas inutile de s'arrêter sur cette réponse, pour voir ce qu'il peut y avoir de contradictoire en lui, ce qui passera par un examen des rapports qu'il peut entretenir avec la question sociale, objet d'un des chapitres les plus longs, et les plus importants du livre.

Pour se faire une idée des confusions qui entourent la notion d'individualisme, rien de mieux que de s'adresser à un de ses plus ardents défenseurs, Hayek. Après bien d'autres, il commence par rappeler la nécessité de distinguer entre un vrai et un faux individualisme. Du premier, nous dit-il, Tocqueville fut un des plus éminents représentants au XIXe siècle ; mais c'est pour ajouter que l'auteur de La démocratie en Amérique utilise le mot individualisme pour désigner une attitude qu'il déplore et rejette ${ }^{1}$. Ainsi, quand le «bon »

${ }^{1}$ F. Hayek, Individualism : True and False (1945), in Studies on the Abuse and Decline of Reason: Text and Documents, The Collected Works of F. A. Hayek, vol. 13, Chicago, 
individualiste parle de l'individualisme, c'est pour en dire du mal! On voit bien que chacun a tendance à mettre un peu ce qu'il veut sous ce mot. Parmi ces multiples usages, une première tentative de clarification invite à en distinguer trois, sans compter l'individualisme méthodologique, dont il n'y a pas lieu de parler ici.

Avant de les examiner, il convient toutefois de souligner le caractère polémique du mot. Ses connotations négatives font que les partisans de l'individualisme préfèrent souvent se présenter comme des libéraux. Qui en effet oserait parler contre la liberté ? Au moment d'aborder le thème, ils sont sur la défensive (la distinction signalée à l'instant relève de cette stratégie) et soulignent la nécessité de commencer par lever les préjugés qui pèsent sur l'individualiste. Parmi ceux-ci, le plus tenace est celui qui identifie individualisme et égoïsme. Nous devons nous défaire une fois pour toutes de l'idée que l'individualiste serait un égoïste ${ }^{2}$ mais, ce point négatif étant acquis, reste à dire ce qu'est l'individualisme.

Une première réponse consiste à y voir le libre développement de l'individualité. Il correspondrait alors à cet idéal de Bildung qui a trouvé son expression classique chez Humboldt et qui a profondément marqué le Mill de On Liberty. On pourrait encore y rattacher la conception de Hayek pour qui l'individualiste, prenant acte de ce que « il n'y a d'échelles de valeurs que partielles, [...] conclut qu'il faut laisser l'individu, à l'intérieur de limites déterminées, libre de se conformer à ses propres valeurs plutôt qu'à celles d'autrui $»^{3}$. Dans les deux cas, le lien avec la liberté, et par ce biais avec une certaine vision de la politique, est explicite. Hayek ne se reconnait toutefois pas dans ce qu'il appelle « l'individualisme allemand ». Accorder une trop grande valeur aux personnalités originales constitue en effet une menace potentielle pour le bon fonctionnement d'une société et il félicite les Anglais d'avoir su se garder ce danger en reconnaissant l'utilité sociale du conformisme ${ }^{4}$

Dans la pensée contemporaine, cette première approche est complétée, et même assez largement supplantée, par une autre, empruntée aux économistes, et dont on trouve une assez bonne expression chez les utilitaristes. L'individu n'y est pas seulement posé comme l'atome de la société, celui par qui et pour qui celle-ci existe. Elle a en propre de reposer sur une théorie de la motivation presque totalement absente dans l'approche précédente. Mandeville avait affirmé que, dans la sphère économique, il existe une harmonie naturelle des intérêts. L'individu n'avait donc plus à se soucier de l'intérêt commun. Il pouvait se livrer sans arrière-pensée à la poursuite de son seul intérêt, la main invisible se chargeant de régler les différents problèmes qui pouvaient surgir. De là cet appel à l'homo oeconomicus, agent rationnel qui cherche à maximiser son profit. De là aussi la morale des utilitaires qui n'est, si on en croit Élie Halévy, que « leur psychologie économique mise à l'impératif $»^{5}$. - C'est sur cette version que s'appuient ceux qui veulent identifier individualisme et égoïsme. Comme le notait le même Halévy, on ne comprendrait rien au programme des utilitaristes « si l'on ne voit pas, d'abord et avant tout, dans leur philosophie pratique, une tentative pour discréditer l'abnégation et réhabiliter l'égoïsme » ${ }^{6}$. Mais l'objection ne tient pas car, après examen, il apparaît que, dans leur doctrine « l'égoïsme de l'individu est à la fois explicitement affirmé et implicitement nié » ${ }^{7}$.

\footnotetext{
University of Chicago Press, p. 49-51.

${ }^{2}$ Hayek, La route de la servitude. Paris: Quadrige/PUF, 2005, p. 49.

${ }^{3}$ Ibid.

${ }^{4}$ Hayek, Studies on the Abuse and Decline of Reason: Text and Documents, The Collected

Works of F. A. Hayek, vol. 13, Chicago, University of Chicago Press, p. 68-69.

${ }^{5}$ Élie Halévy, Le radicalisme philosophique, Paris, PUF, ***, t. 3, p. 206.

${ }^{6}$ Op. cit., p. 201.

${ }^{7}$ Op. cit., p. 237.
} 
Hayek, conscient de la difficulté, était, par des voies différentes, arrivé à la même conclusion.

Alors que cette interprétation tend à faire de l'individualisme une constante de la nature humaine, comme s'il était inscrit dans nos gènes, il en existe une autre, sociologique et non plus économique, qui y voit au contraire le produit d'une évolution sociale. Elle se rattache à l'idée de société des individus, dont il n'est en quelque sorte qu'un sous produit : L'individualisme est la mentalité prédominante dans une telle société. Il peut prendre plusieurs formes et par exemple, même s'il ne s'y reconnaît pas, l'utilitarisme utilitaire est redevable de ce type d'explication. Une des versions les plus connues est sans doute celle de Tocqueville, qui y voyait la situation de l'individu après la chute de l'Ancien Régime : dégagé des liens sociaux constitutifs de la féodalité, l'individu se repliait sur lui-même. Adversaire déclaré de l'utilitarisme, Durkheim avait une toute autre interprétation : pour lui, l'individualisme était une sorte de religion de l'humanité, dont les principes avaient été posés par Rousseau et Kant, et que sa doctrine l'obligeait à considérer comme un produit social.

Le caractère approximatif de cette tentative de classification est manifeste, un même penseur pouvant adopter tour à tour, ou même simultanément, l'une ou l'autre de ces conceptions. Mais déjà on peut se demander : de ces individualismes, quel est celui qui engendre des contradictions, si contradiction il y a ? Sont-elles d'ailleurs toutes issues du même individualisme? Durkheim, par exemple, avait bien pris soin d'expliquer que sa position n'était pas contradictoire : on peut être individualiste, insistait-il, tout en disant que l'individu est un produit de la société. Il entendait par là se prémunir contre l'objection rencontrée plus haut : si l'individualisme est un produit de la dissolution des liens sociaux, par un effet d'entrainement, il peut aussi accélérer le processus et devenir ainsi une menace pour la société. Il faut donc chercher la contradiction dans une autre direction. Pour Olivier Rey, elle tient à la singularité absolue revendiquée par, ou pour, l'individu. Celle-ci échappant à toute prise, il n'y a pas d'autre issue que d'ignorer ce qui le singularise. Déjà, Tocqueville notait que « chaque citoyen, devenu semblable à tous les autres, se perd dans la foule $»^{8}$. Étant celle de tout un chacun, cette revendication n'a de toute façon plus rien de singulier. Elle appelle donc un traitement indifférencié et purement quantitatif, puisque en fin de compte chaque individu en vaut un autre. Pris dans son ensemble l'ouvrage invite toutefois à compléter cette explication par une autre, qui met plus précisément en cause la conception économico-utilitariste de l'individualisme.

L'essor de la société des individus était en effet accompagné d'un phénomène sans précédent. Il y a toujours eu des pauvres, mais les conditions de vie des ouvriers dans la première moitié du dix-neuvième siècle posaient un défi tel qu'il a fallu inventer un nouveau mot pour le nommer : le paupérisme. Au lieu de l'abondance que les progrès de l'industrie étaient censés apporter, pour une grande partie de la population, il n'y avait que misère ; au lieu de l'épanouissement de l'individu, la dégradation. A Londres, l'espérance de vie avait diminué de deux ans. Comment expliquer une situation si contraire aux principes établis? Aujourd'hui où l'on ne parle plus de question sociale, mais de questions sociétales, comme si « social» était devenu un gros mot, les pages que lui consacre Olivier Rey comptent à mon sens parmi les plus intéressantes du livre. A les lire, on ne peut en effet s'empêcher d'établir un parallèle avec la situation actuelle. Les débats qui faisaient rage alors en Angleterre autour de la loi sur les pauvres, que ses adversaires présentaient comme un archaïsme faisant obstacle à la création d'un marché du travail, font étrangement penser aux débats d'aujourd'hui sur le code du travail et la protection sociale. C'est dans ce contexte qu'il faut situer l'œuvre de Malthus. De ce dernier, on ne retient

\footnotetext{
${ }^{8}$ A. de Tocqueville, La démocratie en Amérique, Livre II, 4 e partie, chap. 2, in Cuvres, I 2 , Paris, Gallimard, 1951, p. 298.
} 
d'ordinaire que son influence sur Darwin ; mais c'est oublier qu'il a exercé une influence toute aussi grande sur Ricardo, et par lui sur le développement de la pensée économique. Le succès prodigieux rencontré par son Essai sur la population vient de ce qu'il apportait, à la question : d'où viennent les pauvres ? la réponse attendue par les partisans de l'individualisme utilitaire. Ce n'était pas, comme certains le pensaient, le produit d'une mauvaise organisation sociale, mais plus simplement de lois naturelles auxquelles il n'y avait qu'à se plier.

Si le lien avec les statistiques est ici moins immédiat que dans le premier type d'explication, il reste bien attesté. Galton prolonge Malthus. Dans les deux cas, ce qui est en cause dans notre rapport ambivalent au nombre, ce n'est pas tant les statistiques, dont les services rendus, quand elles sont bien employées, sont incontestables, que l'individualisme. "Si l'on estime que le nombre prend trop de place, c'est moins à lui qu'il faut s'en prendre qu'à la situation qui lui fait aujourd'hui la part si belle » (p. 298), conclut Olivier Rey, qui nous invite ainsi à faire retour sur nous-mêmes et à nous interroger sur le type de société dans lequel nous souhaiterions vivre. 